\section{Primary pancreatic extraskeletal osteosarcoma in a domestic shorthair cat previously diagnosed with diabetes mellitus}

\section{Katarzyna Smiejan, Jon Hall, Jocelyn Bisson, Naomi Earley, Jessica McCarthy, Sionagh Smith, Spela Bavcar}

Hospital for Small Animals, Royal (Dick) School of Veterinary Studies, University of Edinburgh, Edinburgh, United Kingdom

\section{OBJECTIVES}

To describe the clinical signs, clinical pathology, imaging findings, surgical treatment and histopathological findings of a primary pancreatic extraskeletal osteosarcoma, arising from the left limb of the pancreas in a domestic shorthair cat previously diagnosed with diabetes mellitus.

\section{METHODS \\ Case report of one cat.}

\section{RESULTS}

A 12-year-old male neutered domestic shorthair cat presented with acute onset weight loss and hyporexia, as well as chronic onset abdominal distention. The patient was diagnosed with diabetes mellitus two years previously. A large ill-defined ventrocentral abdominal mass and peritoneal effusion were diagnosed using contrast enhanced computed tomography. Neither metastases nor primary bone disease were detected on review of computed tomography images. At exploratory laparotomy the mass originated from the left limb of the pancreas with adhesions to multiple abdominal organs. Excisional biopsy of the mass by left partial pancreatectomy and concurrent splenectomy were performed. Histopathology was consistent with primary pancreatic extraskeletal osteosarcoma. The patient developed cardiopulmonary arrest 3 hours postoperatively and the owners elected for euthanasia.

\section{STATEMENT (CONCLUSIONS)}

To the authors' knowledge, this is the first reported case of a primary pancreatic extraskeletal osteosarcoma in a cat. The connection between the reported extraskeletal osteosarcoma and diabetes mellitus is interesting in this case and, although it might be coincidental, neoplasia of the pancreas has been reported in pancreatic exocrine dysfunction, therefore, extraskeletal osteosarcoma should be considered as a differential diagnosis in feline pancreatic neoplasia.
Intra-abdominal Extraadrenal Paragangliomas with Spinal Cord Invasion in a Siberian Husky Dog: Case Report

\section{Somporn Techangamsuwan ${ }^{1,2}$,} Nicha Hansinlawat ${ }^{2}$, Katriya Chankow ${ }^{2}$, Suwicha Chuthathep ${ }^{3}$, Wijit Banlunara ${ }^{2}$

1 Companion Animal Cancer Research Unit, Bangkok, Thailand

2 Department of Pathology, Faculty of Veterinary Science

Chulalongkorn University, Bangkok, Thailand

3 KB Pet Hospital, Bang Bon, Bangkok, Thailand

\section{OBJECTIVES}

Since paragangliomas in dogs occur rarely in the retroperitoneal or intra-abdominal paravertebral area. We speculated whether this perirenal mass in a dog was the extra-adrenal paraganglioma, investigative techniques were performed for final diagnosis.

\section{METHODS}

A 1-year-old Siberian husky was referred to the hospital with history of back pain and progressive posteriorparalysis. Obvious signs showed generalized weakness and muscle atrophy without pain reflex in both hindlimbs. Magnetic resonance imaging (MRI) of vertebrae and ultrasound-guided cytology were done. Finally, carcass was necropsied after death. Pathological and immunohistochemical (IHC) stainings for neuron specific enolase (NSE), synaptophysin, Vimentin, S100, chromogranin A CDX-2, somatostatin were investigated.

\section{RESULTS}

Cytology suggested malignant mesenchymal tumor. MRI showed a large invasive mass located ventrally to the T11-L3 vertebrae with multiple metastatic lesions to thoracolumbar vertebrae and spinal cord compression. Upon necropsy, a $17 \times 10 \times 8 \mathrm{~cm}$, intra-abdominal, firm mass was observed within the perirenal area. Adrenal glands were easily dissected and not attached to the mass.

Histologically, mass architecture was multilobulated, well-demarcated, and highly cellular. Neoplastic cells 
were polygonal shape arranged in nests, packets, and cords of epithelial cells often forming glandular structures, supported by fibrovascular stroma. Cytomorphology contained distinct cell borders and finely granular eosinophilic cytoplasm. Initial diagnosis was neuroendocrine tumor.

Further IHC diagnosis was done, neoplastic cells revealed strong intracytoplasmic expression of synap- tophysin and NSE. Immunoreactivity to others was negative.

\section{STATEMENT (CONCLUSIONS)}

Based on all findings, intra-abdominal extra-adrenal paraganglioma was diagnosed. Although this tumor is rare in dogs, it should be included in the list of differential diagnosis.
Neoplastic Pericardial Effusion and Widespread Intrathoracic Metastasis in a Dog with a High-Grade Cutaneous Mast Cell Tumour: First Description

\section{Andy Yale ${ }^{1}$, Balazs Szladovits ${ }^{1}$, Anneliese Stell ${ }^{1}$, Scott Fitzgerald ${ }^{2}$, Simon Priestnall ${ }^{1}$, Alejandro Suarez-Bonnet ${ }^{1}$}

1 Royal Veterinary College, London, United Kingdom

2 Michigan State University, Michigan, USA

\section{OBJECTIVES}

To present the first case report of a neoplastic pericardial effusion, and widespread intrathoracic metastasis, secondary to a high-grade cutaneous mast cell tumour in a dog.

\section{METHODS}

An 8-year-old neutered male French Bulldog was presented with a two-day history of intermittent vomiting, reduced appetite, and recent rapid development of multiple cutaneous masses over the head and neck region. Mild tachypnoea and dyspnoea were evident on presentation.

\section{RESULTS}

A moderate volume pericardial and bilateral pleural effusion was confirmed ultrasonographically. Echocardiography demonstrated irregular, heterogeneous thickening of the right ventricular and right atrial free walls, consistent with infiltrative intramyocardial disease. Fine needle aspirates and cytology of one of the cutaneous masses confirmed a mast cell tumour. Pericardial fluid cytology was consistent with a haemorrhagic neoplastic effusion due to mast cell neoplasia.

Histopathology and immunohistochemistry of tissues obtained post-mortem confirmed a high-grade cutaneous mast cell tumour with metastasis to the heart, pericardium, mediastinum, and spleen. No metastatic disease was detected in the submandibular lymph nodes and liver. Immunohistochemical analysis yielded a KIT staining-pattern 2, strong and high Ki67 nuclear labelling (average of 65 cells/grid), and an average of 3 positive AgNORs/nucleus in neoplastic cells. PCR for the activating mutation in exons 8 and 11 of $c$-kit were negative.

\section{STATEMENT (CONCLUSIONS)}

To the authors' knowledge, this is the first report describing a high-grade cutaneous mast cell tumour with widespread intra-thoracic metastasis and neoplastic pericardial mast cell effusion in a dog. 